\title{
Variability of the solar spectral irradiance and energetic particles
}

\author{
Adriana Silva-Válio ${ }^{1}$ \\ ${ }^{1}$ CRAAM, Mackenzie University, São Paulo, Brazil \\ email: avalio@craam.mackenzie.br
}

\begin{abstract}
The total spectral irradiance of the Sun is seen to vary on many time scales. Three timescales are more prominent: (1) the longest one of about 11 years; (2) an intermediate timescale of the order of a few weeks; and (3) the shortest variation from hours to seconds. Every 11 years, the total solar irradiance periodically shows intervals of great activity and periods of almost no activity. The peak to peak variability, however, is less than $0.1 \%$. This periodic variation of 11 years is called the solar cycle, which main tracer are sunspots. During times of maximum activity, there are many sunspots on the surface of the Sun, whereas during minimum there may be none. Sunspots are dark, and therefore cool, regions of enhanced magnetic fields of about a few hundred Gauss, that usually appear in groups on the solar photosphere. Basically, the solar cycle is regulated by the magnetic dynamo acting below the solar surface. Right now, the Sun is going through a time of minimum activity. The sunspot lifetime is of the order of one to two weeks, and are thus responsible for the intermediate variability timescale. The magnetic loops seen in ultraviolet and X-ray images have their footpoints anchored on sunspots. The most energetic phenomena of solar activity are flares and coronal mass ejections. Flares are large explosions that occur on the solar atmosphere and may last from a few seconds to hours. A solar flare is caused by a sudden, and yet unpredicted, energy release high above the magnetic loops. This magnetic energy is then used into particle acceleration and heating of the surrounding atmosphere. Both the energetic particles and the hot gas produce emission throughout the whole electromagnetic spectrum, from the very energetic gamma-rays all the way to long radio waves. From the observation of the emission produced during flares it is possible to infer the energetic particles spectra and thus have a clue on the acceleration mechanism that produced these particles. The recent findings of flare observations at gamma-rays by the RHESSI satellite and at high radio frequencies by the Solar Submillimeter Telescope are presented and discussed.
\end{abstract}

Keywords. Sun: activity, Sun: flares, Sun: particle emission

\section{Solar activity}

The solar variability can occur on three timescales: (1) Solar dynamo: is the longest timescale of about 11 years; (2) Solar rotation and sunspot lifetime: an intermediate timescale of the order of a few weeks; (3) Flares and coronal mass ejections: the shortest timing, varying from hours to seconds.

Sunspots are dark regions seen in solar images in visible light, where high concentration of magnetic fields are located (Figure 1). Because magnetic fields hamper the energy transfer to the photosphere from the convective zone below, these regions end up being cooler than the surrounding photosphere $\left(T_{\text {eff }}=5780 \mathrm{~K}\right)$, with temperatures of about 4000 - $5000 \mathrm{~K}$.

Since 165 B.C. there have been reports of sunspots sighting (Wittmann \& Xu 1987) by the Chinese with the naked eye during sunsets and sunrises when the Sun was close to the horizon, and its brightness was not so intense. Nevertheless, it was only with the first 


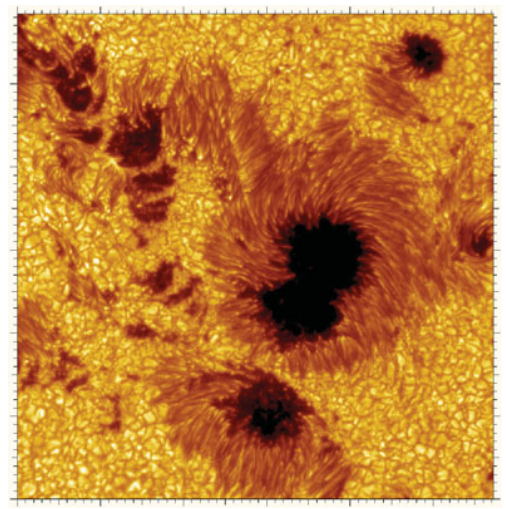

Figure 1. Sunspot observed with the Swedish Solar Telescope (SST). Credit: The SST is operated by the Royal Swedish Academy of Sciences,

observation through a telescope by Galileo in 1610 that sunspots have been monitored regularly. In 1843, Schwabe noticed that the number of sunspots varied periodically every 11 years (Schwabe 1844). This can be seen in the left panel of Figure 2, where the number of sunspots is plotted over the last four centuries.

The solar activity cycle lasts for approximately 11 years, when during periods of maximum activity the Sun is covered with spots. Not only does the sunspot number vary with a period of 11 years, but also the soft X-ray brightness of the Sun, as can be seen from the composite of solar images by the Yohkoh satellite taken every 6 months (right panel of Figure 2). Presently, in 2009, the Sun is still displaying minimum activity.
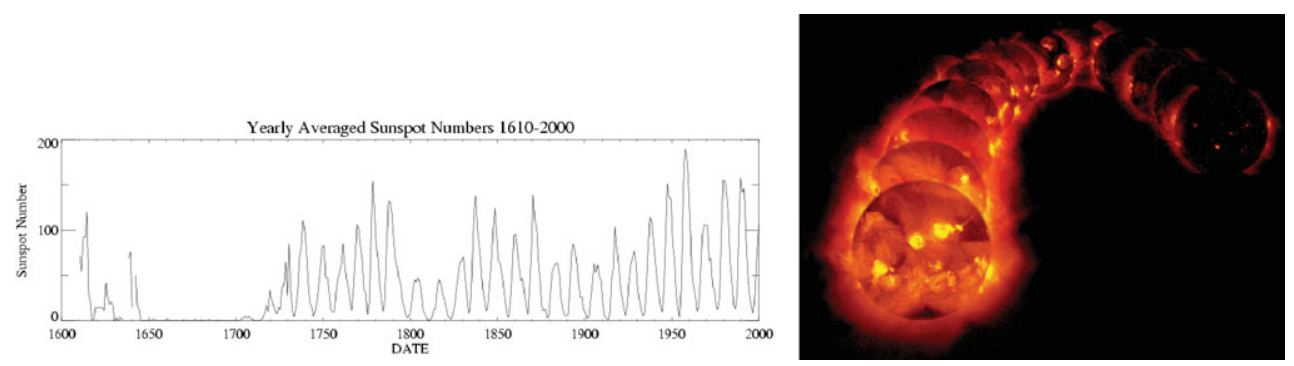

Figure 2. Left: The 11-year periodicity in the Sun activity is easily observed in the sunspot number (http://www.physics.unlv.edu/ jeffery/astro/sun/sun.html) Right: Solar activity cycle seen in the global soft X-ray emission from the Yohkoh satellite.

The solar flux above the Earth's atmosphere, also known as the solar constant, was measured by several satellites. The result of the observations after cross calibration is plotted in Figure 3. As can be seen from the figure, the solar constant is not so constant, but varies by about $0.1 \%$ with an 11 year period, the variation of the total solar output being in phase with the solar cycle. This is at first sight contradictory since during periods of maximum activity there are more sunspots, or dark regions, on the solar surface. However, the plages that are bright regions around sunspots are also more abundant during maximum activity periods and their brightness more than counterbalances the dimming caused by the spots.

Other phenomena also follow the 11 year cycle, such as solar flares, filament eruption, and coronal mass ejections. Flares and mass ejections are the most energetic events in 


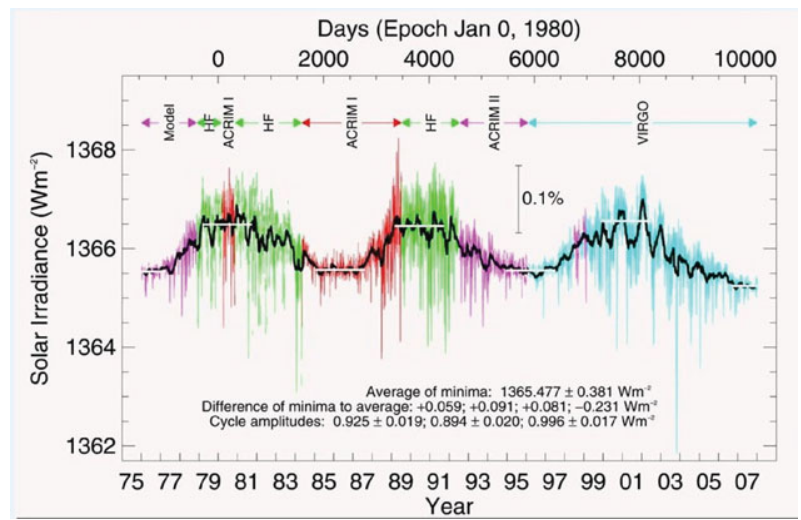

Figure 3. The solar irradiance, or flux at the top of Earth atmosphere, for the past three decades. The data were collected from different satellites and cross calibrated.

the Solar System, and are powered with the magnetic energy contained in the magnetic field loops of the solar atmosphere.

Coronal mass ejections are huge bubbles of solar plasma and magnetic fields that are ejected toward the interplanetary medium during the course of many hours. Some of these mass ejections are directed at Earth and may cause many disturbances once the particles hit the Earth's magnetosphere and atmosphere generating geomagnetic storms, power outages, satellite damage, and auroras, among others. Figure 4 illustrates the mass being ejected from the Sun (left panel) and reaching the Earth magnetosphere (right panel).
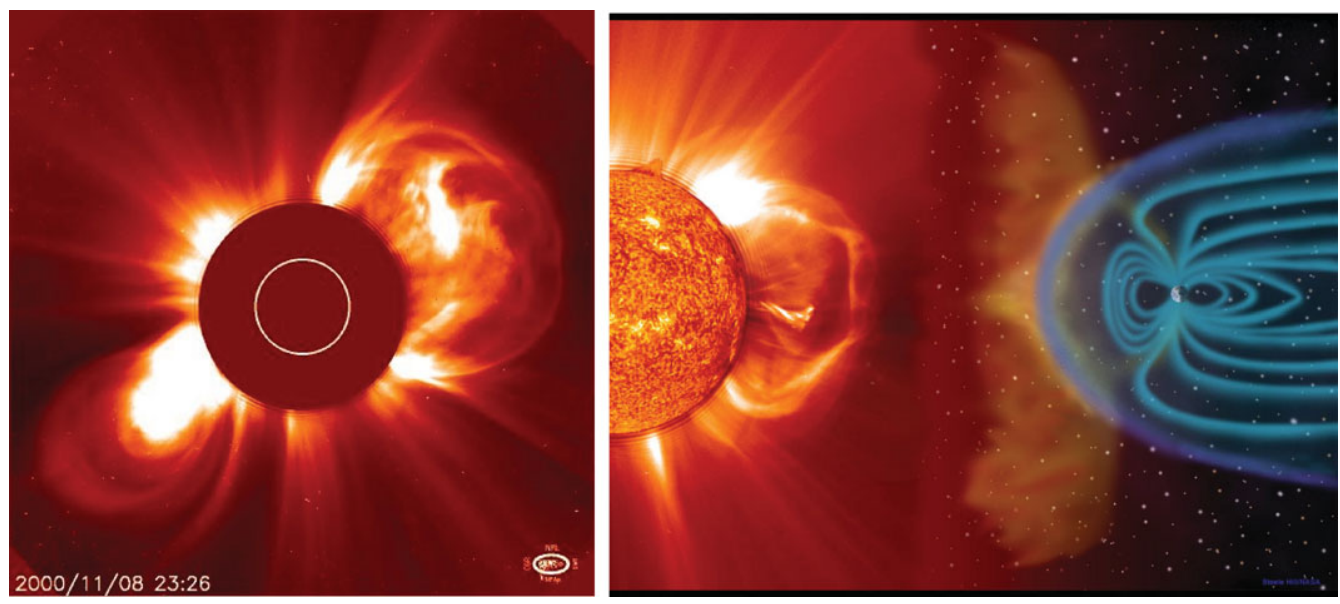

Figure 4. Left: Image of a coronal mass ejection from the Sun observed by the coronograph LASCO on board the SOHO satellite. The occulted solar disc is depicted by the white circle. Right: Illustration of a mass ejection from the Sun and its interaction with the Earth magnetosphere (distances not to scale).

During a solar flare, up to $10^{25} \mathrm{~J}$ of energy is released in only $100-1000 \mathrm{~s}$. Flares always occur within active regions. Filaments (or prominences when seen on the limb) are cold material (5000-10000 K) suspended by magnetic fields. In response to a magnetic instability, they can be suddenly thrown upward. A coronal mass ejection (CME) may throw up to $5 \times 10^{13} \mathrm{~kg}$ of plasma into interplanetary medium at velocities of $200-2000 \mathrm{~km} / \mathrm{s}$, totaling $10^{23}-10^{24} \mathrm{~J}$ of energy. What all these phenomena have in common is that their energy source originates from the reconnection of magnetic field lines. Flares and CMEs 
also show a periodicity of 11 years, occurring more frequently and with greater intensity during periods of solar maximum.

\section{Solar flares}

A solar flare may be thought of as a sudden release of large quantities of energy in timescales of seconds to minutes. The energy source comes from the magnetic field loops located in active regions. This energy then heats the local plasma and accelerates particles up to very high energies (electrons up to $\sim 100 \mathrm{MeV}$ and ions to $\sim 10 \mathrm{GeV}$ ). Both the hot plasma and the energetic particles produce large quantities of radiation throughout the whole electromagnetic spectrum (radio, visible, ultraviolet, X-rays)

An idealized picture of a solar flare has emerged from the observations at all wavelengths of the electromagnetic spectrum. The idealized flare scenario is that magnetic reconnection occurs above the loop top accelerating the particles (see Figure 5). A fraction of these energetic particles escapes toward the interplanetary medium through open field lines, whereas the remaining particles are injected into the loop below. Some of them may become trapped, spiraling around the magnetic lines and thus producing high frequency radio emission by gyrosynchrotron. The ones that are not trapped, or when these trapped particles finally overcome the magnetic bottle through collisions, reach the denser chromosphere below and interact with the ions creating hard X- and gamma-rays. The energy deposited close to the loop footpoints then heats the chromospheric plasma that expands filling the magnetic loop and emitting copious amounts of soft X-ray.
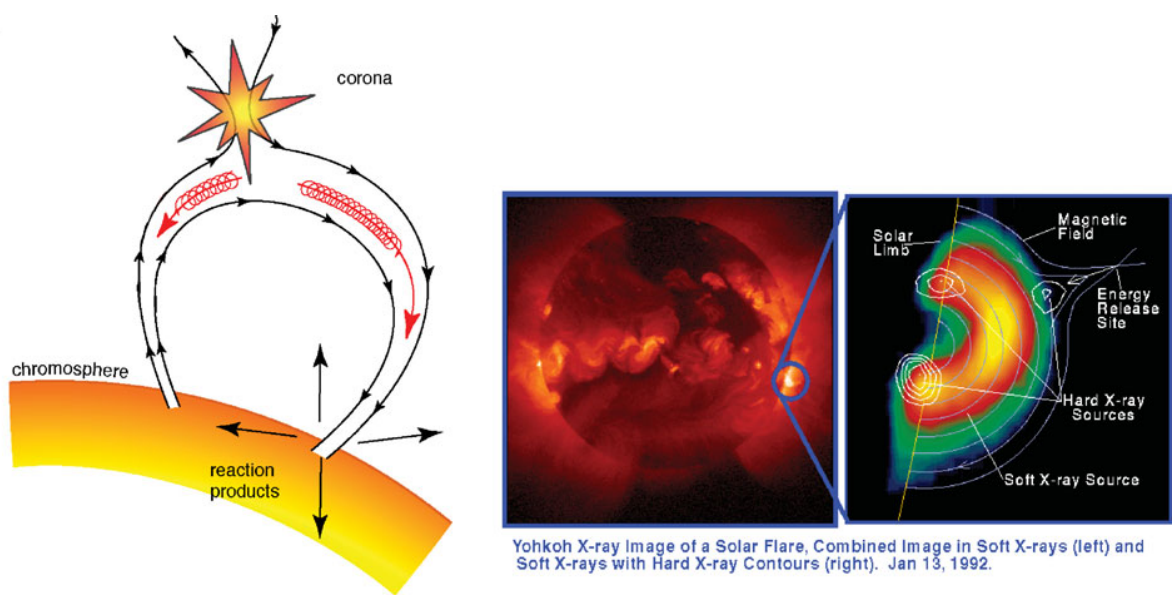

Figure 5. Left: Idealized magnetic loop where a flare occurs. Right: A hard X-ray source detected by the Yohkoh satellite above the loop top for a limb event.

An observational evidence of such model was obtained from the detection of a hard X-ray source above the magnetic field loops for a limb flare (Masuda et al. 1994). The magnetic field loop is that outlined from the low energy soft X-rays, whereas the footpoints are seen as hard X-ray sources (white countours in the left panel of Figure 5). The novelty of this observation is the detection of a hard X-ray source above the loop top at the location where the energetic particles, accelerated at the magnetic reconnection site, were injected downward into the loop and interacted with the plasma within the looptop 
yielding the hard X-ray source by bremsstrahlung. The response of the chromosphere to a flare can also be seen in $\mathrm{H}-\alpha$ images (Figure 6 , left panel).

It is clear from the model depicted above that flares generate emission at all wavelengths of the electromagnetic spectrum. Nevertheless, the temporal evolution is different for each wavelength as shown in the right panel of Figure 6 . The time profile of flare emission can be divided into an impulsive phase due to the accelerated particles and a gradual one created from the thermal plasma. The impulsive peak is clearly seen at high frequency $(>1 \mathrm{GHz})$ radio emission and hard $\mathrm{X}$-rays, whereas the gradual phase is best seen in soft X-rays. The next subsections depict the main discoveries obtained at hard X-ray and submillimeter wavelengths that are thought to be produced by the same population of accelerated particles.
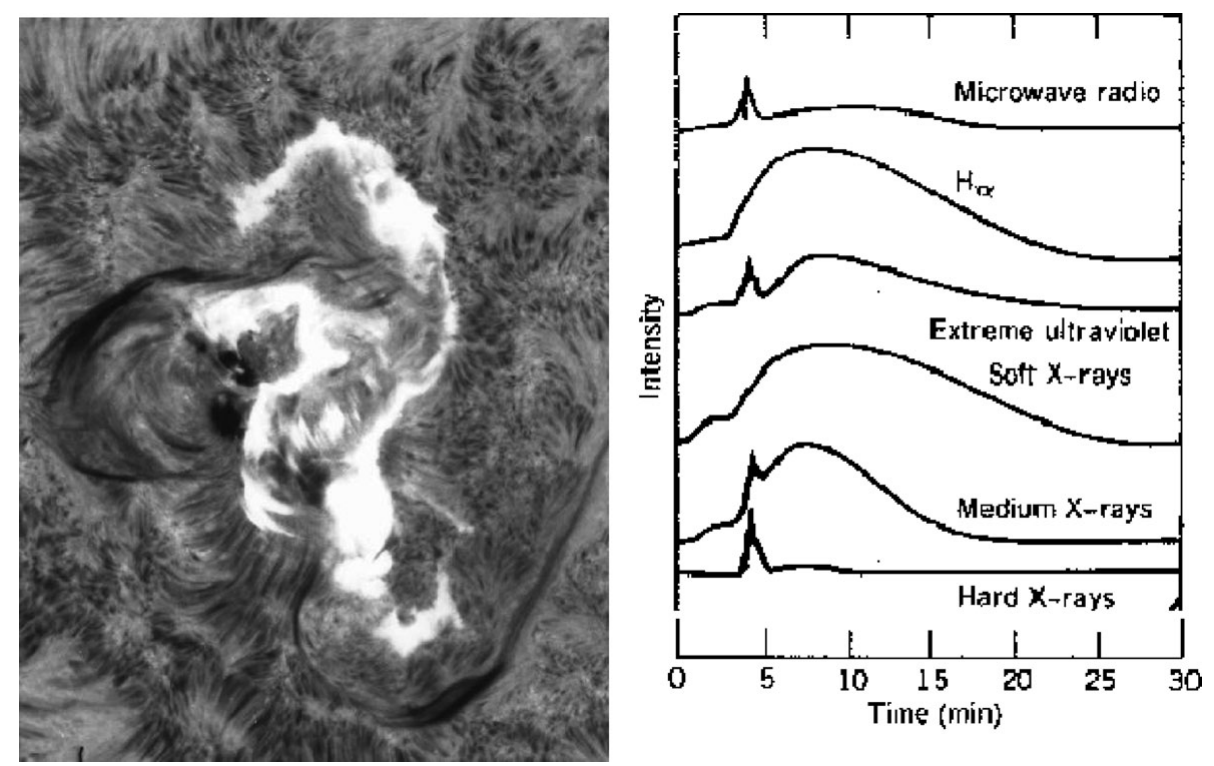

Figure 6. Left: Image of a solar flare through a H- $\alpha$ filter centered at a wavelength of $6563 \AA$ from the Bib Bear Solar Observatory. Right: Time profiles of solar flare emission at several wavelengths showing the initial impulsive phase and later on the more gradual emission.

\subsection{Main discoveries of flares at hard X-rays}

Many new results were obtained with the Reuven Ramaty High Energy Solar Spectroscopic Imager (RHESSI) (Lin et al. 2002). The goal of this solar dedicated satellite, launched on February 2nd, 2002, was to explore the physics of particle acceleration mechanism and energy release in solar flares. Hard X- and gamma-ray images with high spatial (>2"), spectral (1-5 keV), and temporal (10 ms) resolution can be obtained. The spectral range of the observations varies from $3 \mathrm{keV}$ to $15 \mathrm{MeV}$. Among the main discoveries are the first image of gamma-rays from a solar flare and the resolved gamma-ray lines of Fe, $\mathrm{Mg}, \mathrm{Si}, \mathrm{Ne}, \mathrm{C}$, and O. A complete issue of the Astrophysical Journal Letters (volume $595)$ was dedicated to the results obtained from the observations of the flare on July 23, 2003 (Lin et al. 2003).

Gamma-ray lines are produced by nuclear collisions of energetic ions with the solar atmosphere. This is in agreement with the simple flare loop model described above. The detected line centered at an energy of $2.223 \mathrm{MeV}$ is due to Deuterium formation by neutron capture. The left panel of Figure 7 shows the centroid of the $2.223 \mathrm{MeV}$ emission 
detected from the July 23, 2003 flare (Hurford et al. 2003). This flare emission from an ion was found to be shifted by 20" from the lower energy sources at 0.05-1.4 MeV, that are due to electron bremsstrahlung. This implies a different acceleration and/or propagation mechanisms for electrons compared to that of ions in solar flare.

Other gamma-ray lines produced by the de-excitation of ions (C, O, Ne, Mg, Si, Fe) were resolved for the first time and are shown in the right panel of Figure 7 (Smith et al. 2003). The measured lines display a redshift of $0.1 \%-0.8 \%$, and this redshift is seen to decrease with the ion mass. This redshift is larger than that expected for an isotropic distribution of ions in a radial magnetic field. The explanation for the measured redshift is that the magnetic field lines are inclined by $40^{\circ}$ with respect to the solar surface.
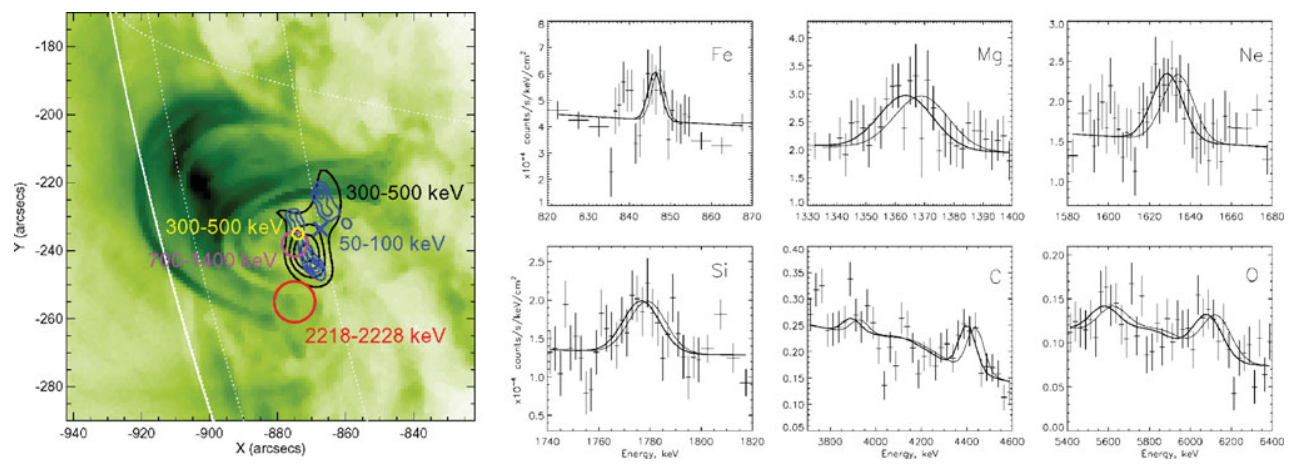

Figure 7. Left: First image at gamma-rays showing that the gamma-ray source at $2.223 \mathrm{MeV}$, due to ions, occurs at a different location than the lower energy sources $(50-300 \mathrm{keV})$ produced by electrons. Right: Resolved gamma-ray lines for six different species of ions.

\subsection{Flare at submillimeter wavelengths}

On the other end of the spectrum is the emission at submillimeter wavelengths. Nevertheless, this emission is thought to be produced by $>\mathrm{MeV}$ electrons (White \& Kundu 1992), the same ones that generate the $100-1000 \mathrm{keV}$ hard X-rays. The results described here were obtained with the Solar Submillimeter Telescope (Figure 8), a $1.5 \mathrm{~m}$ dish installed at the CASLEO Observatory in the Argentinean Andes at an altitude of $2500 \mathrm{~m}$ (Kaufmann et al. 2001). It is a solar dedicated instrument operating with 4 receivers at $212 \mathrm{GHz}$ and 2 receivers at $405 \mathrm{GHz}$, with sub-second temporal resolution.

The SST observed the greatest explosion ever recorded, a GOES class X28, on November 4, 2003 (Kaufmann et al. 2004). This limb event is shown in the left panel of Figure 9, where the white crosses represent the beams of the telescope. The data showed a striking new result, that the flux at $405 \mathrm{GHz}$ was higher than that at $212 \mathrm{GHz}$. This can be seen in the temporal evolution of the submm emission plotted in the middle panel of the figure, where 4 main peaks can be seen. Radio spectra taken at the time of the first (P1) and fourth (P4) peaks were obtained by combining the SST data with microwave data from the Owens Valley Solar Array (OVSA). The spectra plotted in the right panel of Figure 9 clearly show that the submm data points at 212 and $405 \mathrm{GHz}$ do not follow the microwave spectral decrease but rather increase toward higher frequencies. This new spectral component has been named the "THz component" because the peak of the spectrum is expected to be located somewhere in the $\mathrm{THz}$ domain.

The November 4, 2003, flare was one of the so called Halloween events and was not the only flare observed to display an increasing spectral component at submm wavelengths. Three other flares showed the same increasing spectra at frequencies $>200 \mathrm{GHz}$ : the 


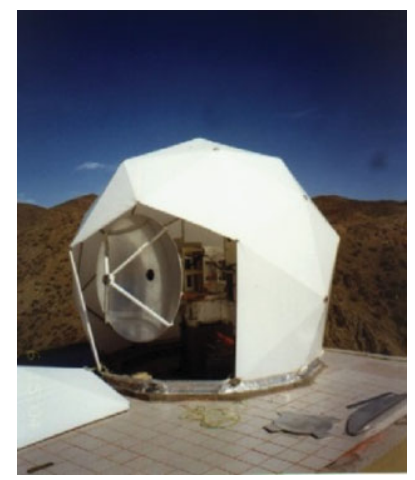

Figure 8. The Solar Submillimeter Telescope, a solar dedicated radio antenna installed in Argentina operating at 212 and $405 \mathrm{GHz}$.

GOES X2.0 flare on April 12, 2001 (Lüthi, Magun, \& Miller 2004b) and the GOES X17.2 flare on October 28, 2003 (Lüthi, Lüdi, \& Magun 2004a) were both observed by the KOSMA submillimeter telescope at 230 and $345 \mathrm{GHz}$. The other flare, a GOES X8.7 on November 2, 2003, occurred on the same active region as the one on November 4th, was the first flare detected simultaneously by SST and RHESSI (Silva et al. 2007).
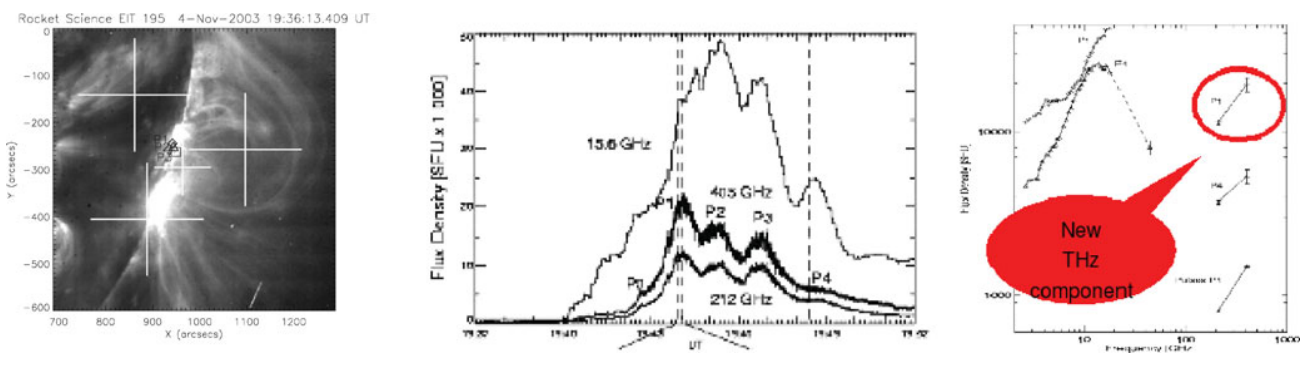

Figure 9. The November 4, 2003. Left: The gray scale shows the ultraviolet emission from the flare, whereas the symbols represent the centroid of $212 \mathrm{GHz}$ emission at different times. The white crosses correspond to the size of SST beams. Middle: Temporal evolution of the November 4, 2003, flare at $15 \mathrm{GHz}$ and submm wavelengths. Right: Radio spectra of the flare combining the microwaves from OVSA and the submm data from SST. A new spectral component peaking somewhere in the $\mathrm{THz}$ is depicted.

The flare emission was located mainly in the magnetic loop outlined in ultraviolet light shown in Figure 10 (left panel), where the contour levels represent the hard X-rays at several energies and the centroid of the submm emission is depicted by the small black points. As expected, the high energy hard X-rays are located close to the loop footpoints. The temporal evolution of the flare emission at several wavelengths are plotted in the middle panel of Figure 10.

Two impulsive peaks are seen in the microwaves and $<500 \mathrm{keV}$ emission. The submm flux also displays the second impulsive peak (the first peak occurred during a calibration scan of the telescope) and a long duration emission that lasts for several minutes. This gradual emission was also detected at greater than $2 \mathrm{MeV}$ hard X-rays. This good correlation implies that the submm emission is in fact associated to the high energy electrons $(>5 \mathrm{MeV})$ that also produce the high energy hard X-rays.

The radio spectra of this flare, composed of microwave data from OVSA and submm emission observed by the SST, are plotted in Figure 10 (right panel) for nine time intervals. Again the THz spectral component is evident with the flux at $405 \mathrm{GHz}$ even higher 

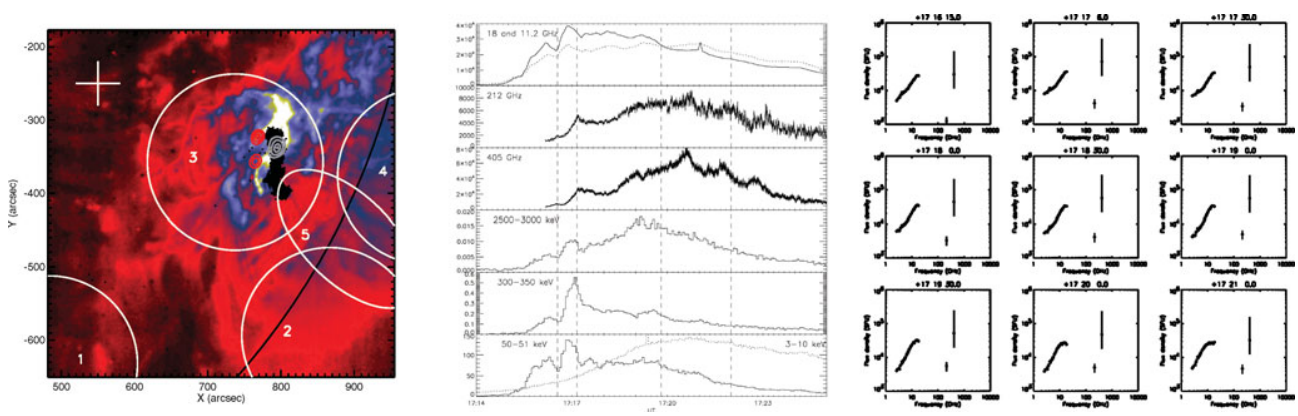

Figure 10. The November 2, 2003, flare. Left: The gray scale corresponds to the ultraviolet emission of this event, whereas the superimposed contours represent the hard X-ray emission $(12-25 \mathrm{keV}$ in gray and $100-300 \mathrm{keV}$ in red. The black points are the centroid position of the $212 \mathrm{GHz}$ emission, where the cross mark the absolute uncertainty in position. The white circles show the SST beams $(1,2,3$, and 4 at $212 \mathrm{GHz}$, and 5 is the $405 \mathrm{GHz}$ beam). Middle: Time profiles of the flare emission at microwaves from OVSA, submm waves from SST, and hard X-rays from RHESSI. Right: Radio spectra composed by microwave and submm data of the flare at nine time intervals.

than that at microwaves. Silva et al. (2007) analyzed three possible emission mechanisms in order to unravel the nature of this novel spectral component. The authors propose three possibilities for the submm source: (1) Free-free emission from thermal electrons; gyrosynchrotron radiation from (2) positrons or from (3) non-thermal electrons.

If the increasing spectral component at frequencies larger than $200 \mathrm{GHz}$ is due to bremsstrahlung from thermal electrons, then the submm emission could have been produced by a 20 " source of temperature $\sim 10^{7} \mathrm{~K}$ and densities of $10^{12}-10^{14} \mathrm{~cm}^{-3}$, shown as a dashed line in the left panel of Figure 11 (for a full discussion, see Silva et al. 2007). The soft X-ray flux that would have been produced by such source was calculated (Thomas, Crannel, \& Starr 1985) and is about a 100 times larger than the soft X-rays observed by the GOES satellite.

The second possibility is that the submm emission originates from positrons as they gyrate around the magnetic field lines. Positrons are believed to be produced by pion or radioactive decay after nuclear interactions during a large flare. RHESSI detected the 511-keV line due to the electron-positron annihilation (Share et al. 2004). The number of positrons estimated from the annihilation-line fluence detect by RHESSI, however, is 3000 to $2 \times 10^{5}$ times smaller than that need to account for the submm flux. Thus this explanation can also be ruled out.

When considering gyrosynchrotron emission from non-thermal electrons it is necessary to match simultaneously the submm flux and the hard X-rays since both types of emission are believed to be produced by the same population of energetic electrons. The hard Xray spectra from RHESSI yield $5 \times 10^{35}$ electrons between $50 \mathrm{keV}$ and $20 \mathrm{MeV}$ and an electron spectral distribution of index $\delta=2.5$ above $\sim 3 \mathrm{MeV}$. Using this same electron spectra to fit the submm emission, Silva et al. (2007) found that this required a very compact source of 0.5 " radius, with high density $\left(10^{12} \mathrm{~cm}^{-3}\right)$ in a region of very strong magnetic field $(4500 \mathrm{G})$. This same electron spectrum can also model the microwave spectra $(1-15 \mathrm{GHz})$ as produced by a more extended source of 50 " radius in a $480 \mathrm{G}$ magnetic field region. An example of the gyrosynchrotron fits (Ramaty 1969, Ramaty et al. 1994) to the radio spectra at the time of maximum submm flux are shown as solid lines in Figure 11 (left panel). 

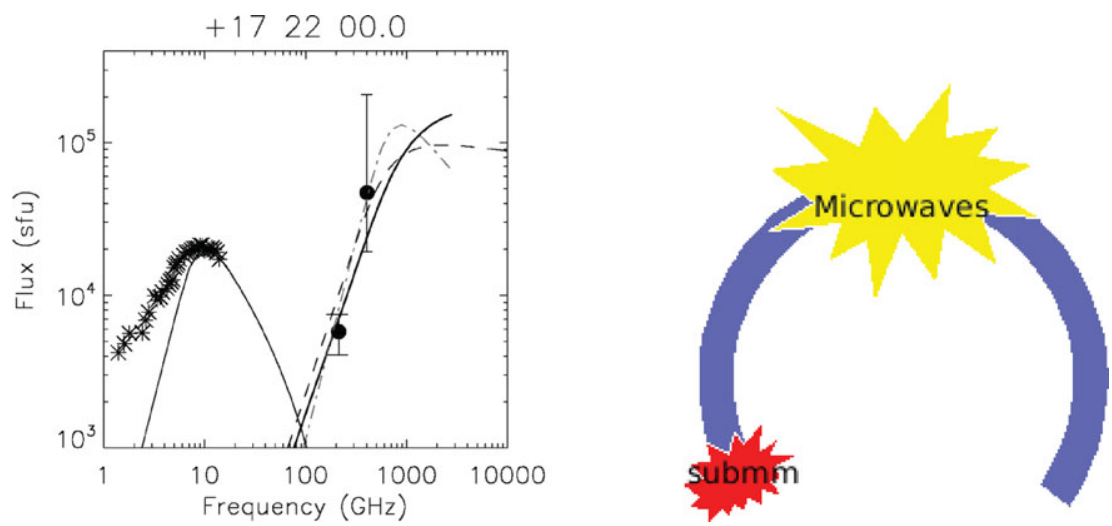

Figure 11. Left: Radio spectra at the time of the $405 \mathrm{GHz}$ peak emission with the fitting results for different emission mechanisms: bremsstrahlung from thermal electrons (dashed line), gyrosynchrotron from positrons (dot-dashed curve) and non-thermal electrons (solid line). Right: Cartoon depicting the microwave loop top source and the submm source at the footpoint.

\section{Conclusions}

The Sun despite being considered a quiet star when compared to others is still the source of very energetic events such as solar flares and coronal mass ejections. Since the XIX century mainly from the monitoring of sunspots on its surface, it is known that the Sun follows an 11 year cycle. Actually, the true solar cycle due to the magnetic dynamo has a duration of 22 years, when the global magnetic field returns to its original polarity. Sunspots are the main indicators of solar activity, but the occurrence of flares and CMEs is also observed to have an 11 year periodicity.

The analysis of the flare emission at several wavelengths is crucial for an understanding of the global picture of a solar flare that follows. A solar flare is powered by the magnetic energy released in magnetic field reconnection above looptops. This energy then goes into particle acceleration, both electrons and ions, and plasma heating. A fraction of the energetic particles is injected into the magnetic loop below. Some of them may become trapped and emit radio waves by gyrosynchrotron, while the ones that escape the magnetic trap reach the loop footpoints below and produce hard X- and gammarays as a result of the interaction with the denser material of the chromosphere. All this input of energy heats the plasma that then expands filling the loop, this is known as "chromospheric evaporation" and the whole heated loop at temperatures of tens of millions of $\mathrm{K}$ radiates in soft $\mathrm{X}$-rays and radio wavelengths.

With the launch of the RHESSI satellite in 2002 and the installation of the SST in 1999, many interesting results have been found about solar flares. A very well studied flare that occurred on July 23, 2003, provided new insight into the physics of gamma-ray production. For the first time, an image of a source of ion emission was obtained and showed that possibly a different acceleration mechanism or distinct propagation paths from that of the accelerated electrons is at work. Gamma-ray lines displayed redshifts that corroborated the picture of down streaming ions into the chromosphere during a flare and determined the inclination of magnetic loops with respect to the solar surface.

Observations of solar flares at high radio frequencies have uncovered a new spectral component that is likely to peak in the $\mathrm{THz}$ domain. Four flares observed with two different radio telescopes (SST and KOSMA) have shown an increasing emission toward higher frequencies $(>200 \mathrm{GHz})$. Here I have highlighted the main results of the flares that occurred on November 4th (Kaufmann et al. 2004) and 2nd (Silva et al. 2007). 
Simultaneous observations of the November 2, 2003, flare in hard X-rays and radio waves allowed the conclusion for the best explanation of this new spectral component. The data were best fit as produced by emission of a single population of non-thermal electrons with a total number of $5 \times 10^{35}$ and a power-law index of $\delta=2.5$ above $\sim 3 \mathrm{MeV}$. These energetic electrons are responsible for producing the hard X-rays by bremsstrahlung and the radio waves by gyrosynchrotron in two distinct emitting regions. The microwaves originate from the same electrons trapped in a low density $\left(10^{9} \mathrm{~cm}^{-3}\right)$ region, 100" in size, located higher in the loop where the magnetic field strength is $480 \mathrm{G}$. On the other hand the submillimetric source was very small (size of 1 ") and located at the loop footpoints in a region of high density (accelerated electron densities of $10^{12} \mathrm{~cm}^{-3}$ ) and strong magnetic fields $(B=4500 \mathrm{G})$. Both the microwave and the submillimeter sources are depicted in the cartoon of Figure 11 (left panel).

\section{References}

Hurford, G. J., Schwartz, R. A., Krucker, S., Lin, R. P., Smith, D. M., \& Vilmer, N. 2003, ApJ (Letters), 595, 77

Kaufmann, P., Costa, J. E. R., Giménez de Castro, C. G., Hadano, Y. R., Kingsley, J. S., Kingsley, R. K., et al. 2001, In: J. J. Pinho, G. P. S. Cavalcante, \& A. H. Oliveira (eds.), Proc. 2001 SBMO/IEEE MTT-S International Microwave and Optoeletronics Conference, p. 439

Kaufmann, P., Raulin, J.-P., de Castro, C. G. G., Levato, H. Gary, D. E., Costa, J. E. R., et al. 2004, ApJ (Letters), 603, 121

Lin, R. P., Dennis, B. R., Hurford, G. J., Smith, D. M., Zehnder, A., Harvey, P. R., et al. 2002, Solar Phys., 210, 3

Lin, R. P., Krucker, S., Hurford, G. J., Smith, D. M., Hudson, H. S., Holman, G. D., Schwartz, R. A., Dennis, B. R., Share, G. H., Murphy, R. J. et al. 2003, ApJ (Letters), 595, 69

Lüthi, T., Lüdi, A., \& Magun, A. 2004a, $A \& A$, 420, 361

Lüthi, T., Magun, A., \& Miller, M. 2004b, A\&A, 415, 1123.

Masuda, S., Kosugi, T., Hara, H., Tsuneta, S., \& Ogawara, Y. 1994, Nature, 371, 495

Ramaty, R. 1969, ApJ, 158, 753

Ramaty, R., Schwartz, R. A., Enome, S., \& Nakajima, H. 1994, ApJ, 436, 941

Schwabe, M. 1844, Astronomische Nachrichten, 21, 223

Share, G. H., Murphy, R. J., Smith, D. M., Schwartz, R. A., \& Lin, R. P. 2004, ApJ (Letters), 615,169

Silva, A. V. R., Share, G. H., Murphy, R. J., Costa, J. E. R., de Castro, C. G. Gimnez, Raulin, J.-P., \& Kaufmann, P. 2007, Solar Phys., 245, 311

Smith, D. M., Share, G. H., Murphy, R. J., Schwartz, R. A., Shih, A. Y., \& Lin, R. P. 2003 ApJ (Letters), 595, 81

Thomas, R. J., Crannell, C. J., \& Starr, R. 1985, Solar Phys., 95, 323

White, S. M. \& Kundu, M. R. 1992, Solar Phys., 141, 347

Wittmann, A. D. \& Xu, Z. T. 1987, A\&A Suppl. Ser., 70, 83 